\title{
OPEN The impact of aspirin on Klebsiella pneumoniae liver abscess in diabetic patients
}

\author{
Chien-Hsiang $\mathrm{Tai}^{1}{ }^{1}$, Chien-Ning Hsu ${ }^{2,3}$, Shih-Cheng Yang ${ }^{4}$, Cheng-Kun $\mathrm{Wu}^{4,5}$, \\ Chih-Ming Liang ${ }^{4,5}$, Wei-Chen Tai ${ }^{4,5}$, Seng-Kee Chuah ${ }^{4,5}$ \& Chen-Hsiang Lee ${ }^{1,5 \bowtie}$
}

In this study, we aimed to investigate the impact of aspirin on the risk of pyogenic liver abscess caused by Klebsiella pneumoniae (KP-PLA) and invasive KP-PLA syndrome (IKPS) in diabetic patients. Diabetic patients who were propensity-score matched were retrospectively included from hospital-based database. Kaplan-Meier approach with a log-rank test was used to compare the cumulative incidences of KP-PLA including IKPS between aspirin users and non-users. Totally, 63,500 patients were analyzed after propensity-score matching (1:1). Compared with that of non-users, the incidence of KP-PLA was significantly reduced in aspirin users $(0.31 \%$ vs. $0.50 \%, p<0.01)$, but not for that of IKPS $(0.02 \%$ vs. $0.03 \%, p=0.29$ ). Patients taking aspirin for $\geq 90$ days had a significantly lower risk for KP-PLA (hazard ratio, $0.67 ; 95 \% \mathrm{Cl}, 0.50-0.90)$. Females, taking clopidogrel or metformin for $\geq 90$ days, and taking $\mathrm{H} 2$-blockers or proton pump inhibitors (PPIs) for $\geq 5$ days were also associated with a lower risk of KP-PLA. However, cholangitis and a glycated hemoglobin $\geq 8.5 \%$ were associated with an increased risk of KP-PLA.

Pyogenic liver abscess (PLA), an infection of the liver parenchyma, is a potentially life-threatening disease, the incidence of which is increasing in many countries such as the US, Denmark and Taiwan ${ }^{1-3}$. Diabetes mellitus (DM), liver cirrhosis, and advanced age are known risk factors for PLA ${ }^{4-6}$. Among these at-risk individuals, diabetic patients tend to have more serious complications of PLA, for which Klebsiella pneumoniae (KP) has been shown to be the leading pathogen ${ }^{7,8}$. The majority of KP isolates that cause PLA (KP-PLA) are of K1 and K2 capsule serotypes, which account for $60-70 \%$ and 5-20\% of the cases reported in Southeast Asia, respectively ${ }^{9-11}$. Invasive KP-PLA syndrome, including PLA and metastatic infections involving unusual distant sites such as the eye, brain, lung, or prostate, have also been observed in diabetic patients, especially in those with poor glycemic control $^{12}$.

Nuclear factor $\mathrm{\kappa B}$ signaling and the interleukin-12/interferon-gamma signaling pathway are disrupted in diabetic animals and patients, which might play an important regulatory role in the development of KP-PLA ${ }^{13,14}$. Furthermore, in-vitro studies revealed that, in high glucose media, KP serotypes K1 increased gene expression and increased synthesis of the capsular polysaccharide (CPS), which is an important virulence factor for $\mathrm{KP}^{15,16}$. It was also observed that phagocytosis of $\mathrm{KP}$ serotypes $\mathrm{K} 1$ and $\mathrm{K} 2$ by neutrophils was impaired in type 2 diabetic patients with poor glycemic control, and this was associated with an increased risk of metastatic complications $^{16,17}$. An unsubstantiated but biologically plausible mechanism for this observation is that metastatic seeding by KP with specific serotypes during bacteremia is due to DM-related loss of vascular patency or integrity.

Therapeutic drugs administered for some chronic diseases may affect the recurrence of PLA in diabetic patients. Our previous study have demonstrated that aspirin could reduce the recurrence rate of PLA ${ }^{18}$. Aspirin can trigger lipoxin and enhances macrophage phagocytosis of bacteria ${ }^{19}$. We previously also reported that aspirin enhanced the susceptibility of KP serotype K1 to leukocyte phagocytosis by reducing CPS production; moreover, our multivariate analysis revealed that patients with community-acquired KP bacteremia who had recently used aspirin were at a lower risk of acquiring invasive KP-PLA syndrome ${ }^{20}$. Beyond the general understanding that $\mathrm{DM}$ is a risk factor for the development of invasive KP-PLA syndrome, little is known about how aspirin affects its development in diabetic patients. To the best of our knowledge, no large-scale population-based study has

\footnotetext{
${ }^{1}$ Division of Infectious Diseases, Department of Internal Medicine, Kaohsiung Chang Gung Memorial Hospital, Kaohsiung, Taiwan. 'Department of Pharmacy, Kaohsiung Chang Gung Memorial Hospital, Kaohsiung, Taiwan. ${ }^{3}$ School of Pharmacy, Kaohsiung Medical University, Kaohsiung, Taiwan. ${ }^{4}$ Division of Hepato-Gastroenterology, Department of Internal Medicine, Kaohsiung Chang Gung Memorial Hospital, Kaohsiung, Taiwan. ${ }^{5}$ College of Medicine, Chang Gung University, Kaohsiung, Taiwan. ${ }^{\square}$ email: lee900@ cgmh.org.tw
} 


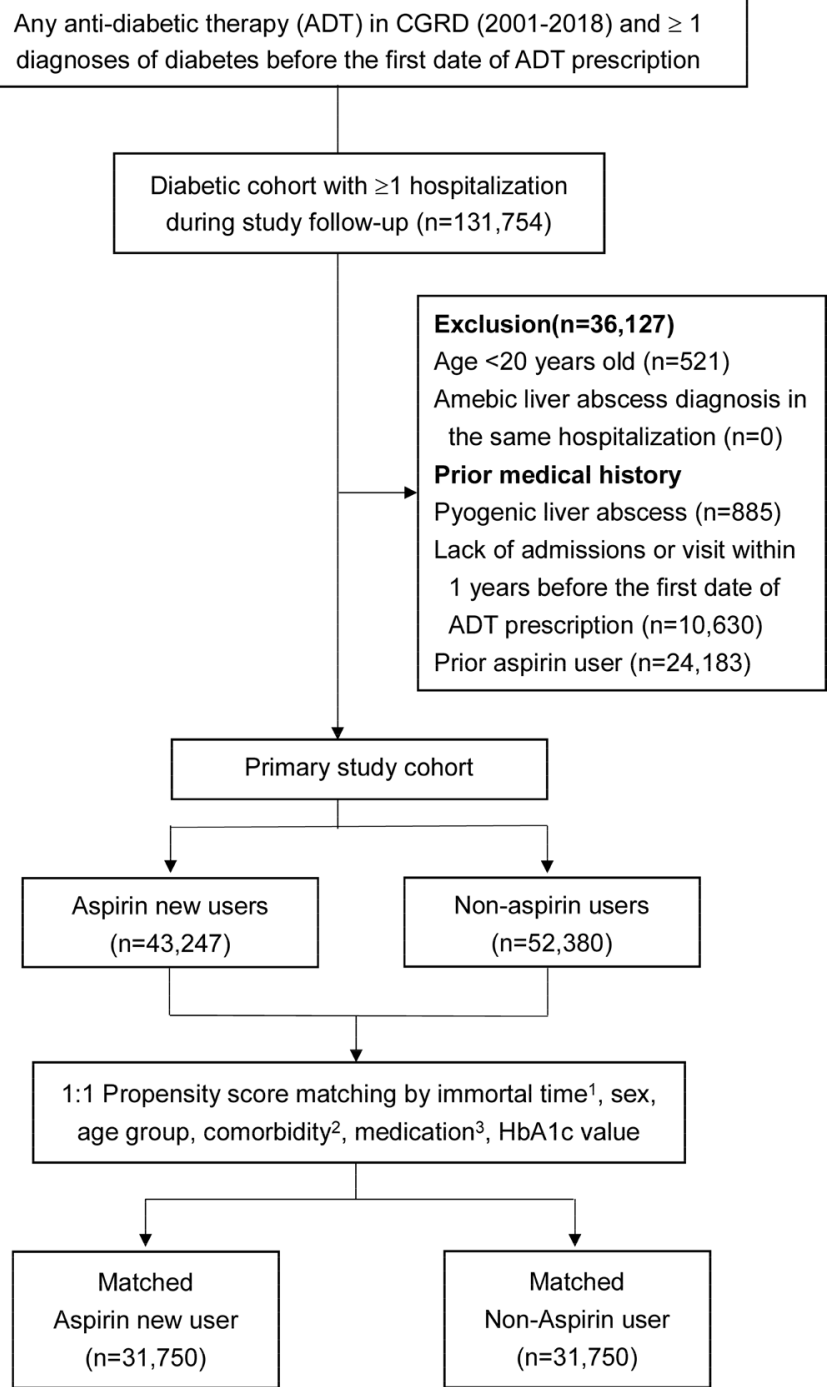

Figure 1. Flow chart of patient selection. 1. Immortal time: Patients who received aspirin were propensity matched in a 1:1 ratio with those who were not taking aspirin and were alive at the time of the first dispensed prescription to their matched counterpart. 2. Comorbidities: cirrhosis, injury to the liver, hepatobiliary malignancy, gastric cancer, colon cancer, cholangitis, bile duct stones/cholelithiasis, viral hepatitis, hepatitis $\mathrm{B}$, hepatitis $\mathrm{C}$, renal disease, hepatocellular carcinoma, metastatic cancers, other malignancies, ischemic heart disease and myocardial infarction, ischemic stroke, arrhythmia, all cardiovascular or cerebrovascular accident events, and atherosclerosis. 3. Medication: centrally acting anti-adrenergic agents, ą-blockers, thiazide-type diuretics, $ß$-blockers, calcium channel blockers, angiotensin-converting enzyme inhibitors, angiotensin II receptor antagonists, anti-diabetic therapies (metformin, sulfonylureas, acarbose, TZD, DPP4, GLP1, SGLT2, meglitinides, and insulin).

previously been performed to investigate the association between KP-PLA, including invasive KP-PLA syndrome, and aspirin use.

The primary aim of the current study was to determine the role of aspirin use in diabetic patients and its association with the risk of KP-PLA and invasive KP-PLA syndrome.

\section{Results}

Clinical characteristics of diabetic patients with and without aspirin use. A total of 95,627 patients diagnosed with diabetes between 2001 and 2018 fulfilled the study criteria (Fig. 1). The mean age was $62.04 \pm 12.65$ years, $48 \%$ of the included patients were female, $1.6 \%$ had cholangitis, $67.4 \%$ took metformin, and the mean value of glycated hemoglobin was $8.42 \pm 2.25 \%$. Table 1 shows their clinical characteristics before and after PSM.

In the PSM cohort, the baseline comorbid conditions and prior medications were not significantly different between the aspirin users and non-users. The mean follow-up time was 5.69 years (Supplementary Table S1-1). Among aspirin users, $14.2 \%$ and $35.8 \%$ were exposed to aspirin for 1-90 days and $>90$ days during the followup, respectively (Supplementary Table S1-2). Table 2 presents the concomitant medications used during the 


\begin{tabular}{|c|c|c|c|c|c|c|c|c|}
\hline & \multicolumn{4}{|c|}{ Unmatched cohort $(n=95,627)$} & \multicolumn{4}{|c|}{ PSM cohort $(n=63,500)$} \\
\hline & $\begin{array}{l}\text { Total }(\mathrm{n}=95,627) \\
\mathrm{n}(\%)\end{array}$ & $\begin{array}{l}\text { Aspirin users } \\
(\mathrm{n}=43,247) \mathrm{n}(\%)\end{array}$ & $\begin{array}{l}\text { Non-users } \\
(n=52,380) \text { n (\%) }\end{array}$ & SMD $^{*}$ & $\begin{array}{l}\text { Total }(n=63,500) \\
n(\%)\end{array}$ & $\begin{array}{l}\text { Aspirin users } \\
(\mathrm{n}=31,750) \mathbf{n}(\%)\end{array}$ & $\begin{array}{l}\text { Non-users } \\
(\mathbf{n}=31,750) \mathbf{n}(\%)\end{array}$ & $\mathrm{SMD}^{*}$ \\
\hline \multicolumn{9}{|l|}{ Sex } \\
\hline Male & $49,118(51.36)$ & $22,505(52.04)$ & $26,613(50.81)$ & 0.02 & $32,157(50.64)$ & $16,151(50.87)$ & $16,006(50.41)$ & 0.01 \\
\hline Female & $46,509(48.64)$ & $20,742(47.96)$ & $25,767(49.19)$ & 0.02 & $31,343(49.36)$ & $15,599(49.13)$ & $15,744(49.59)$ & 0.01 \\
\hline $\begin{array}{l}\text { Age at diabetes diag- } \\
\text { nosis, years }\end{array}$ & & & & 0.16 & & & & 0.03 \\
\hline $20-64$ & $54,508(57.00)$ & $22,796(52.71)$ & $31,712(60.54)$ & & $36,201(57.01)$ & $17836(56.18)$ & $18,365(57.84)$ & \\
\hline$\geq 65$ & $41,119(43.00)$ & $20,451(47.29)$ & $20,668(39.46)$ & & $27,299(42.99)$ & $13,914(43.82)$ & $13,385(42.16)$ & \\
\hline \multicolumn{9}{|c|}{ Baseline comorbid condition } \\
\hline \begin{tabular}{|l|l} 
Liver cirrhosis \\
\end{tabular} & $538(0.56)$ & $78(0.18)$ & $460(0.88)$ & 0.10 & $162(0.26)$ & $75(0.24)$ & $87(0.27)$ & 0.01 \\
\hline Unspecified liver injury & $28(0.03)$ & $6(0.01)$ & $22(0.04)$ & 0.02 & $12(0.02)$ & $6(0.02)$ & $6(0.02)$ & 0.00 \\
\hline $\begin{array}{l}\text { Hepatobiliary malig- } \\
\text { nancy }\end{array}$ & $328(0.34)$ & $46(0.11)$ & $282(0.54)$ & 0.08 & $75(0.12)$ & $44(0.14)$ & $31(0.10)$ & 0.01 \\
\hline Gastric cancer & $419(0.44)$ & $92(0.21)$ & $327(0.62)$ & 0.06 & $166(0.26)$ & $86(0.27)$ & $80(0.25)$ & 0.00 \\
\hline Colon cancer & $1222(1.28)$ & $370(0.86)$ & $852(1.63)$ & 0.07 & $641(1.01)$ & $319(1.00)$ & $322(1.01)$ & 0.00 \\
\hline $\begin{array}{l}\text { Hepatocellular car- } \\
\text { cinoma }\end{array}$ & $2588(2.71)$ & $381(0.88)$ & $2207(4.21)$ & 0.21 & $738(1.16)$ & $374(1.18)$ & $364(1.15)$ & 0.00 \\
\hline Metastatic cancers & $3132(3.28)$ & $364(0.84)$ & $2768(5.28)$ & 0.26 & $647(1.02)$ & $361(1.14)$ & $286(0.90)$ & 0.02 \\
\hline Other malignancies & $9039(9.45)$ & $2232(5.16)$ & $6807(13.00)$ & 0.28 & $3972(6.26)$ & $2065(6.50)$ & $1907(6.01)$ & 0.02 \\
\hline Cholangitis & $1011(1.06)$ & $275(0.64)$ & $736(1.41)$ & 0.08 & $478(0.75)$ & $245(0.77)$ & $233(0.73)$ & 0.00 \\
\hline Cholelithiasis & $3223(3.37)$ & $1180(2.73)$ & $2043(3.90)$ & 0.07 & $1906(3.00)$ & $939(2.96)$ & $967(3.05)$ & 0.01 \\
\hline Hepatitis B & $2562(2.68)$ & $505(1.17)$ & $2057(3.93)$ & 0.18 & $933(1.47)$ & $487(1.53)$ & $446(1.40)$ & 0.01 \\
\hline Hepatitis C & $2623(2.74)$ & $551(1.27)$ & $2072(3.96)$ & 0.17 & $1037(1.63)$ & $522(1.64)$ & $515(1.62)$ & 0.00 \\
\hline Other viral hepatitis & $97(0.10)$ & $27(0.06)$ & $70(0.13)$ & 0.02 & $46(0.07)$ & $22(0.07)$ & $24(0.08)$ & 0.00 \\
\hline Chronic kidney disease & $7530(7.87)$ & $3509(8.11)$ & $4021(7.68)$ & 0.02 & $4957(7.81)$ & $2609(8.22)$ & $2348(7.40)$ & 0.03 \\
\hline \multicolumn{9}{|c|}{ Cardiovascular events } \\
\hline $\begin{array}{l}\text { Ischemic heart disease } \\
\text { and myocardial } \\
\text { infarction }\end{array}$ & $13,632(14.26)$ & $9345(21.61)$ & $4287(8.18)$ & 0.38 & $7548(11.89)$ & $3852(12.13)$ & $3696(11.64)$ & 0.02 \\
\hline Ischemic stroke & $8400(8.78)$ & $5896(13.63)$ & $2504(4.78)$ & 0.31 & $4671(7.36)$ & $2433(7.66)$ & $2238(7.05)$ & 0.02 \\
\hline Arrythmia & $5372(5.62)$ & $2951(6.82)$ & $2421(4.62)$ & 0.09 & $3626(5.71)$ & $1868(5.88)$ & $1758(5.54)$ & 0.01 \\
\hline $\begin{array}{l}\text { Any cardiovascular } \\
\text { or cerebrovascular } \\
\text { accident event }\end{array}$ & $28,975(30.30)$ & $18,226(42.14)$ & $10,749(20.52)$ & 0.48 & $17,760(27.97)$ & $9142(28.79)$ & $8618(27.14)$ & 0.04 \\
\hline Atherosclerosis & $1861(1.95)$ & $1133(2.62)$ & $728(1.39)$ & 0.09 & $1152(1.81)$ & $621(1.96)$ & $531(1.67)$ & 0.02 \\
\hline \multicolumn{9}{|l|}{ Prior medications } \\
\hline \multicolumn{9}{|l|}{ Anti-diabetic therapy } \\
\hline Metformin & $64,474(67.42)$ & $29,688(68.65)$ & $34,786(66.41)$ & 0.05 & $43,382(68.32)$ & $21,612(68.07)$ & $21,770(68.57)$ & 0.01 \\
\hline Sulfonylurea & $49,442(51.70)$ & $24,566(56.80)$ & $24,876(47.49)$ & 0.19 & $33,779(53.20)$ & $16,779(52.85)$ & $17,000(53.54)$ & 0.01 \\
\hline Acarbose & $6637(6.94)$ & $3205(7.41)$ & $3432(6.55)$ & 0.03 & $4489(7.07)$ & $2266(7.14)$ & $2223(7.00)$ & 0.01 \\
\hline Thiazolidinedione & $4730(4.95)$ & $2848(6.59)$ & $1882(3.59)$ & 0.14 & $3164(4.98)$ & $1568(4.94)$ & $1596(5.03)$ & 0.00 \\
\hline DPP4 inhibitor & $4543(4.75)$ & $1637(3.79)$ & $2906(5.55)$ & 0.08 & $2643(4.16)$ & $1398(4.40)$ & $1245(3.92)$ & 0.02 \\
\hline GLP-1 agonist & $11(0.01)$ & $4(0.01)$ & $7(0.01)$ & 0.00 & $7(0.01)$ & $4(0.01)$ & $3(0.01)$ & 0.00 \\
\hline SGLT2 inhibitor & $135(0.14)$ & $43(0.10)$ & $92(0.18)$ & 0.02 & $60(0.09)$ & $39(0.12)$ & $21(0.07)$ & 0.02 \\
\hline Meglitinides & $7584(7.93)$ & $3718(8.60)$ & $3866(7.38)$ & 0.04 & $5177(8.15)$ & $2620(8.25)$ & $2557(8.05)$ & 0.01 \\
\hline Insulins & $2174(2.27)$ & $428(0.99)$ & $1746(3.33)$ & 0.16 & $835(1.31)$ & $423(1.33)$ & $412(1.30)$ & 0.00 \\
\hline $\begin{array}{l}\text { Centrally acting anti- } \\
\text { adrenergic agents }\end{array}$ & $332(0.35)$ & $162(0.37)$ & $170(0.32)$ & 0.01 & $216(0.34)$ & $109(0.34)$ & $107(0.34)$ & 0.00 \\
\hline$\alpha$-blockers & $1255(1.31)$ & $562(1.30)$ & $693(1.32)$ & 0.00 & $793(1.25)$ & $414(1.30)$ & $379(1.19)$ & 0.01 \\
\hline Thiazide-type diuretics & $11,343(11.86)$ & $5609(12.97)$ & $5734(10.95)$ & 0.06 & $7364(11.60)$ & $3674(11.57)$ & $3690(11.62)$ & 0.00 \\
\hline \begin{tabular}{l|l} 
B-blockers \\
\end{tabular} & $18,784(19.64)$ & $10,258(23.72)$ & $8526(16.28)$ & 0.19 & $12,035(18.95)$ & $6037(19.01)$ & $5998(18.89)$ & 0.00 \\
\hline $\begin{array}{l}\text { Calcium channel } \\
\text { blockers }\end{array}$ & $27,997(29.28)$ & $14,595(33.75)$ & $13,402(25.59)$ & 0.18 & $18,935(29.82)$ & $9595(30.22)$ & $9340(29.42)$ & 0.02 \\
\hline $\begin{array}{l}\text { Angiotensin-convert- } \\
\text { ing enzyme inhibitors }\end{array}$ & $10,628(11.11)$ & $6523(15.08)$ & $4105(7.84)$ & 0.23 & $6915(10.89)$ & $3386(10.66)$ & $3529(11.11)$ & 0.01 \\
\hline $\begin{array}{l}\text { Angiotensin II receptor } \\
\text { antagonists }\end{array}$ & $20,967(21.93)$ & $11,678(27.00)$ & $9289(17.73)$ & 0.22 & $14,790(23.29)$ & $7393(23.29)$ & $7397(23.30)$ & 0.00 \\
\hline HbAlc value & $8.42 \pm 2.25$ & $8.40 \pm 2.17$ & $8.43 \pm 2.33$ & 0.26 & $8.45 \pm 2.25$ & $8.46 \pm 2.21$ & $8.45 \pm 2.28$ & 0.03 \\
\hline
\end{tabular}

Table 1. Baseline characteristics before and after propensity score matching. DPP4, dipeptidyl peptidase 4 inhibitors; GLP1, glucagon-like peptide-1 analogues; HbA1c, glycated hemoglobin; SGLT2, sodium-glucose co-transporter 2 inhibitors; SMD, standardized mean differences. ${ }^{\star}$ an $\mathrm{SMD}<0.1$ was considered well balance. 


\begin{tabular}{|l|l|l|l|}
\hline & Aspirin users $(\mathbf{n}=\mathbf{3 1}, \mathbf{7 5 0}) \mathbf{n}(\%)$ & Non-users $(\mathbf{n}=\mathbf{3 1}, \mathbf{7 5 0}) \mathbf{n}(\%)$ & $\boldsymbol{p}$ value \\
\hline Clopidogrel & & & $<0.01$ \\
\hline Non-exposure & $21,308(67.11)$ & $29,350(92.44)$ & \\
\hline $1-90$ days & $3447(10.86)$ & $657(2.07)$ & \\
\hline$>90$ days & $6995(22.03)$ & $1743(5.49)$ & \\
\hline Ticagrelor & & & $<0.01$ \\
\hline Non-exposure & $30,322(95.50)$ & $31,726(99.92)$ & \\
\hline Yes & $1428(4.50)$ & $24(0.08)$ & \\
\hline Other anti-platelet agents & & & $<0.01$ \\
\hline Non-exposure & $28,641(90.21)$ & $30,904(97.34)$ & \\
\hline Yes & $3109(9.79)$ & $846(2.66)$ & \\
\hline H2-blocker or proton pump inhibitors & & & $<0.01$ \\
\hline Non-exposure & $11,951(37.64)$ & $15,304(48.20)$ & \\
\hline $1-4$ days & $1445(4.55)$ & $1380(4.35)$ & \\
\hline $5-30$ days & $3109(9.79)$ & $3334(10.50)$ & \\
\hline $31-90$ days & $3652(11.50)$ & $3425(10.79)$ & \\
\hline$>90$ days & $11,593(36.51)$ & $8307(26.16)$ & $<0.01$ \\
\hline Metformin & & & \\
\hline Non-exposure & $4506(14.19)$ & $5139(16.19)$ & \\
\hline $1-30$ days & $1353(4.26)$ & $2090(6.58)$ & \\
\hline $31-90$ days & $1527(4.81)$ & $2243(7.06)$ & \\
\hline$>90$ days & $24,364(76.74)$ & & \\
\hline & & & \\
\hline
\end{tabular}

Table 2. Concomitant medications used during follow-up in the matched cohort.

study follow-up. Among the aspirin-users, $22.0 \%$ had taken clopidogrel for $>90$ days, 57.8\% H2-blockers or proton pump inhibitors for $>5$ days, and $76.7 \%$ metformin for $>90$ days during the follow-up. There was a small number of patients with unspecified liver injury $(n=28)$, other viral hepatitis $(n=97)$, or taking centrally acting anti-adrenergic agents $(n=332)$ and they were not considered in further analyses.

KP liver abscess and in-hospital mortality. The cumulative incidence of KP-PLA was $0.35 \%$ in the primary cohort and $0.41 \%$ in the PSM cohort over the 15 years of follow-up. In the primary cohort, the incidence of KP-PLA was significantly higher in the non-user group compared with the aspirin user group $(0.57 \% v s .0 .29 \%$, $p<0.01$ ) (Table 3). This significant difference in the incidence of KP-PLA remained between the two groups after matching $(0.50 \%$ in the non-users $v s .0 .31 \%$ in the aspirin users; log-rank test, $p<0.01)$ (Fig. 2 ).

The incidence of invasive KP-PLA syndrome was low $(n=18$ in the primary cohort and $n=14$ in the PSM cohort) and was not significantly different between the aspirin user and non-user groups in both primary and PSM cohorts (Table 3). In-hospital mortality was $9.3 \%$ in the primary cohort and $8.2 \%$ in the PSM cohort over the study period. Although the all-cause mortality rate was slightly higher in the aspirin user group compared with the non-user group $(8.83 \% v s .7 .64 \%, p<0.01)$ in the PSM cohort, it was not significantly different over the study period (log-rank test, $p=0.24$ ) (Fig. 3 ).

Duration of aspirin exposure was significantly associated with the risk of KP-PLA, after controlling for baseline characteristics and concomitant medications during the follow-up (Table 4). The risk of KP-PLA was significantly lower among patients with $>90$ days of aspirin exposure (aHR, $0.67 ; 95 \% \mathrm{CI}, 0.50-0.90 ; p=0.01$ ) compared with non-users; however, there was no significant difference between those with $<90$ days of aspirin exposure (aHR, 0.87; 95\%CI, 0.56-1.34; $p=0.52$ ) and non-users.

Compared with male patients, females were associated with a significantly lower risk of KP-PLA (aHR, 0.58; $95 \% \mathrm{Cl}, 0.45-0.75 ; p<0.01)$ (Table 4). Diabetic patients with cholangitis (aHR, 3.00; 95\%Cl, $1.21-7.41 ; p=0.02$ ), and those who had a poorly controlled glucose status with a glycated hemoglobin value $\geq 8.5 \%$ (aHR, 1.80; $95 \% \mathrm{Cl}, 1.30-2.50 ; p<0.01)$ were associated with a significantly greater risk of developing KP-PLA. In contrast, taking clopidogrel for $>90$ days (aHR, $0.41 ; 95 \% \mathrm{Cl}, 0.22-0.75 ; p<0.01$ ), taking metformin for $>90$ days (aHR, $0.50 ; 95 \% \mathrm{Cl}, 0.35-0.72 ; p<0.01$ ), or taking $\mathrm{H}_{2}$-blockers or proton pump inhibitors for $>5$ days were associated with a significantly reduced risk of developing KP-PLA.

\section{Discussion}

The current study demonstrates that use of aspirin for $>90$ days was associated with a lower risk of KP-PLA in diabetic patients without an increase in mortality. Although the risk of KP-PLA decreased with aspirin use, that of invasive KP-PLA syndrome did not. This was probably due to the low prevalence of metastatic infection in both aspirin users and non-users. Aspirin has been reported to inhibit the production of KP CPS, which is important to its virulence because of its anti-phagocytic effect against macrophages and neutrophils ${ }^{12,21}$. Plateletmonocyte aggregation is also involved in the pathogenesis of KP-PLA in diabetic patients, which was shown to be decreased by aspirin ${ }^{22}$. 


\begin{tabular}{|c|c|c|c|c|c|c|}
\hline & \multicolumn{3}{|c|}{ Unmatched cohort $(n=95,627)$} & \multicolumn{3}{|l|}{ PSM cohort $(n=63,500)$} \\
\hline & $\begin{array}{l}\text { Aspirin users }(n=43,247) \\
n(\%)\end{array}$ & Non-users $(n=52,380) n(\%)$ & $p$ value & $\begin{array}{l}\text { Aspirin users }(\mathrm{n}=31,750) \\
\mathrm{n}(\%)\end{array}$ & Non-users $(n=31,750) n(\%)$ & $p$ value \\
\hline K. pneumoniae liver abscess & $124(0.29)$ & $298(0.57)$ & $<0.01$ & $98(0.31)$ & $159(0.50)$ & $<0.01$ \\
\hline $\begin{array}{l}\text { Invasive K. pneumoniae liver } \\
\text { abscess syndrome }\end{array}$ & $5(0.01)$ & $13(0.02)$ & 0.14 & $5(0.02)$ & $9(0.03)$ & 0.29 \\
\hline \multicolumn{7}{|l|}{ Extrahepatic complications } \\
\hline Endophthalmitis & $5(0.01)$ & $2(0.00)$ & & $5(0.02)$ & $0(0.00)$ & \\
\hline Brain abscess & $0(0.00)$ & $1(0.00)$ & & $0(0.00)$ & $1(0.00)$ & \\
\hline Bacterial meningitis & $0(0.00)$ & $4(0.01)$ & & $0(0.00)$ & $3(0.01)$ & \\
\hline Lung abscess & $0(0.00)$ & $5(0.01)$ & & $0(0.00)$ & $4(0.01)$ & \\
\hline Abscess of the prostate & $0(0.00)$ & $1(0.00)$ & & $0(0.00)$ & $1(0.00)$ & \\
\hline In-hospital mortality & $3976(9.19)$ & $4932(9.42)$ & 0.24 & $2804(8.83)$ & $2426(7.64)$ & $<0.01$ \\
\hline
\end{tabular}

Table 3. Comparisons of incidences of K. pneumoniae liver abscess, invasive K. pneumoniae liver abscess syndrome and mortality between aspirin users and non-users in unmatched cohort and propensity-score matched (PSM) cohort.

Time to liver abscess

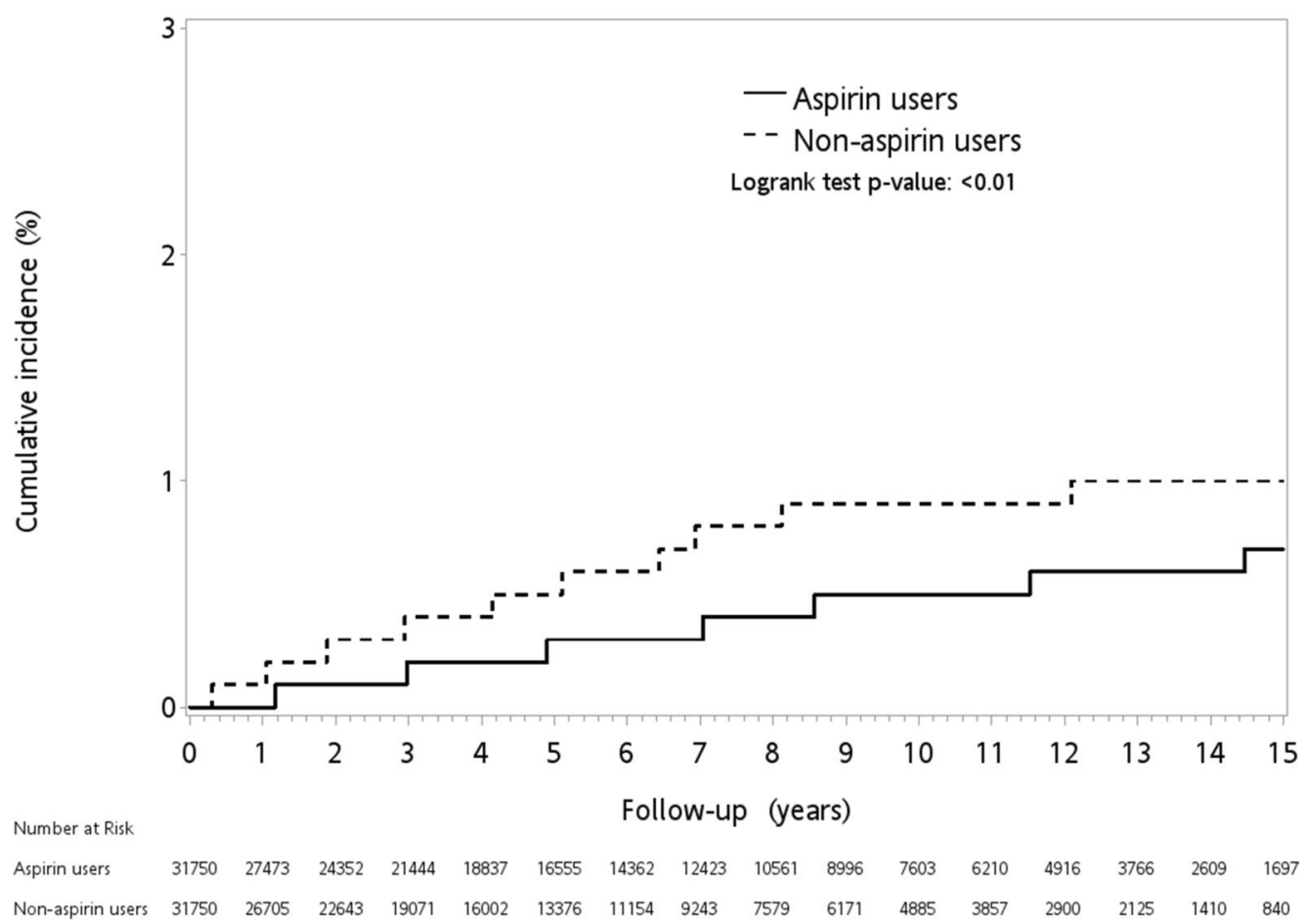

Figure 2. Kaplan-Meier curve of the incidence of Klebsiella pneumoniae liver abscess in diabetic patients with or without aspirin use.

In addition to aspirin use, our study revealed that taking clopidogrel reduced the risk of KP-PLA. Chao et al. found that taking aspirin and clopidogrel for 1-12 months could change the intestinal flora; the number of Lactobacillales and Lactobacillaceae within the flora increased at the order level and family level, respectively ${ }^{23}$. The development of KP-PLA is associated with changes in the gut microbiota, especially a reduction in the Lactobacillales and Lactobacillaceae flora ${ }^{24}$. Some species of Lactobacillus have been proved to inhibit KP in vitro ${ }^{25}$. On the other hand, ticagrelor and other anti-platelet agents, such as dipyridamole, tirofiban, or cilostazol, were not demonstrated to change the gut microbiota. Our study also did not find that these agents were associated with a reduced risk of KP-PLA.

Our study demonstrates that using $\mathrm{H}_{2}$ receptor antagonists or proton pump inhibitors for a minimum of 5 days was associated with a lower risk of KP-PLA. Previous studies had shown that treatment with proton pump inhibitors increased Lactobacillales not only in animal models but also in healthy individuals ${ }^{26,27}$. There was no direct evidence for changes in the gut microbiota after using $\mathrm{H}_{2}$ receptor antagonists, yet it seemed that the microflora 
Time to mortality

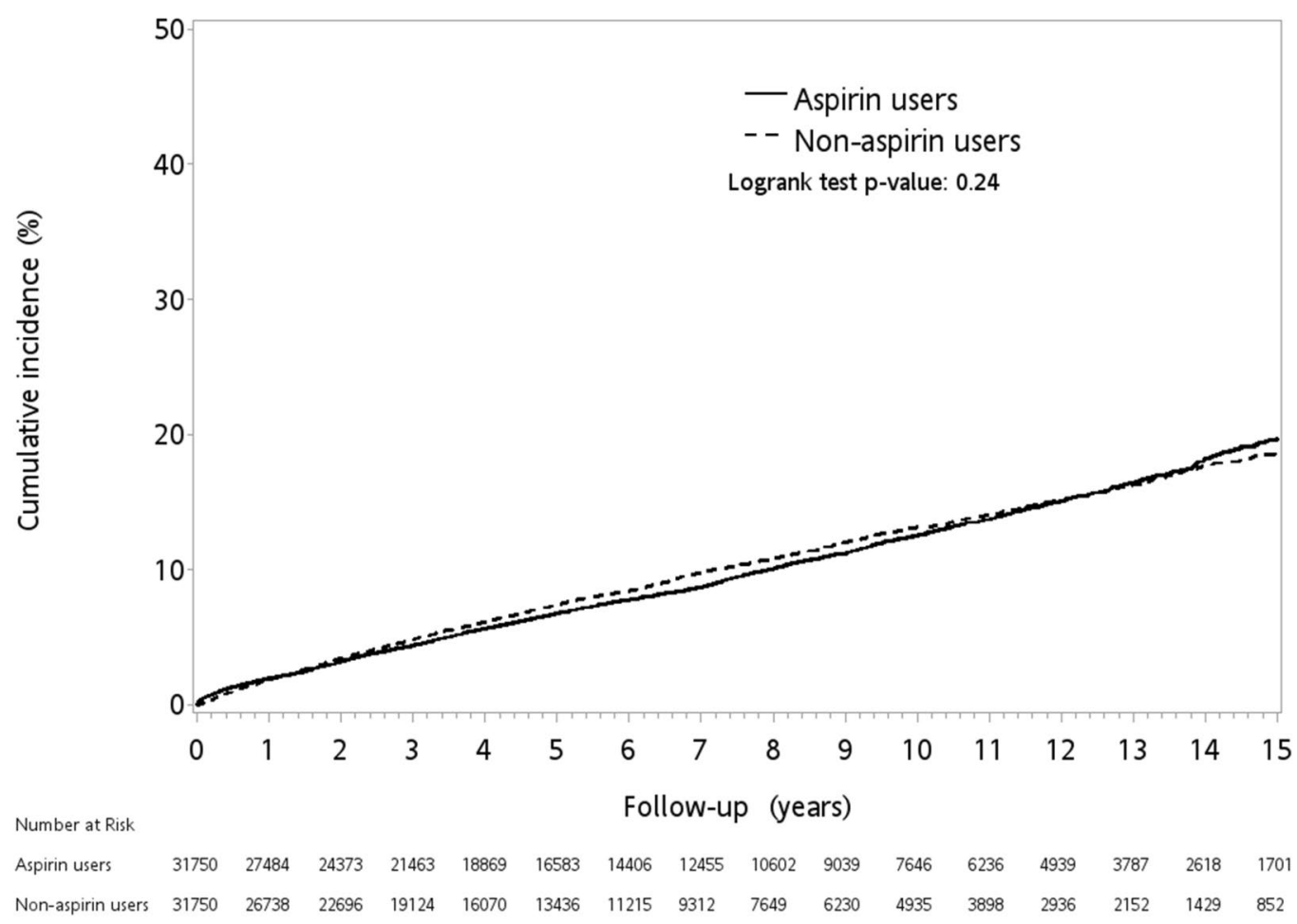

Figure 3. Kaplan-Meier curve of in-hospital mortality in diabetic patients with or without aspirin use.

was still affected by those medications ${ }^{28,29}$. In contrast to our findings, Wang et al. used the National Health Insurance Research Database (NHIRD) in Taiwan and discovered that using proton pump inhibitors for $>5$ cumulative defined daily doses increased the risk of PLA ${ }^{30}$. Our study, using the CGRD, could accurately identify diabetic patients with KP-PLA, and this information cannot be gathered from the NHIRD. After propensity-score matching of the glycated hemoglobin value, we found that using metformin for $>90$ days could considerably reduce the risk of KP-PLA. Individuals taking metformin for 2 months were shown to have changes in their gut microbiota, especially in the growth of Bifidobacterium, of which some strains seemed to have antibacterial activity against enteropathogenic bacteria ${ }^{31,32}$. Other studies revealed increased amount of Lactobacillus with metformin treatment ${ }^{33,34}$. These results indicate that metformin could alter the human gut microbiota; however, how it affects KP colonization in the human gut warrants more investigations.

Compared to male patients, female patients had a lower incidence of PLA, not only in our study but also in previous studies using the Taiwan NHIRD ${ }^{6,18}$. Biliary tract disease was reported to be associated with PLA and was much more likely to be associated with polymicrobial infections, including Escherichia coli, Enterococcus and Klebsiella species ${ }^{6,35}$. Our study supported the finding that diabetic patients who previously had cholangitis were at greater risk of KP-PLA. Patients with a glycated hemoglobin level $>7 \%$ were associated with impaired phagocytosis of KP serotypes K1 and K2 and a higher incidence of PLA ${ }^{17,36}$. Our findings support the theory that poor glycemic control, defined as a glycated hemoglobin level more than $8.5 \%$, was associated with a significantly increased risk of KP-PLA.

A strength of the current study was that it was performed using the CGRD, a database gathered from two medical centers and five local hospitals, with high overall coverage of individuals seeking care reimbursed by the National Health Insurance in Taiwan ${ }^{37}$. It could provide detailed information on the patients' diagnosis, medications, laboratory and examination data. However, there were still some limitations. Previous studies demonstrated that patients in the CGRD had more severe comorbidities than the general population ${ }^{37}$. Therefore, we used propensity-score matching to minimize the differences in comorbidities. In addition, patients might return to their primary care facilities, not the Chang Gung Memorial Hospitals, for their long-term follow-up after their disease stabilized. This might cause bias in the event rate and mortality. To eliminate the bias of loss to follow-up, we excluded patients who didn't have any medical records for one year prior to their diagnosis of diabetes. The incidence of invasive KP-PLA syndrome was previously reported to be $15 \%$ in Taiwan ${ }^{12}$; however, it was about 4.2\% (18/422) and 5.4\% (14/257) in unmatched cohort and PSM cohort, respectively in our study. The prevalence of invasive KP-PLA syndrome might be under-estimated in our study because it was not a routine to screen for any metastatic infection in each patient with KP-PLA in clinical practice. Finally, it was a retrospective study and there might be some confounding factors that were not measured in the analysis despite adjustment by propensity-score matching. 


\begin{tabular}{|c|c|c|c|}
\hline \multicolumn{4}{|l|}{ Aspirin } \\
\hline Non-exposure & \multicolumn{3}{|l|}{ Ref } \\
\hline $1-90$ days & 0.87 & $(0.56-1.34)$ & 0.52 \\
\hline$>90$ days & 0.67 & $(0.50-0.90)$ & 0.01 \\
\hline \multicolumn{4}{|l|}{ Sex } \\
\hline Male & \multicolumn{3}{|l|}{ Ref } \\
\hline Female & 0.58 & $(0.45-0.75)$ & $<0.01$ \\
\hline \multicolumn{4}{|l|}{ Age group } \\
\hline $20-64$ years & \multicolumn{3}{|l|}{ Ref } \\
\hline$\geq 65$ years & 0.89 & $(0.67-1.17)$ & 0.39 \\
\hline \multicolumn{4}{|l|}{ Comorbidities } \\
\hline Liver cirrhosis & 2.44 & $(0.31-18.93)$ & 0.39 \\
\hline Hepatobiliary malignancy & 2.88 & $(0.38-21.80)$ & 0.31 \\
\hline Gastric cancer & 2.24 & $(0.31-16.19)$ & 0.42 \\
\hline Colon cancer & 0.47 & $(0.07-3.41)$ & 0.46 \\
\hline Hepatocellular carcinoma & 2.08 & $(0.73-5.96)$ & 0.17 \\
\hline Metastatic cancers & 0.81 & $(0.11-6.14)$ & 0.84 \\
\hline Other malignancies & 0.88 & $(0.47-1.63)$ & 0.68 \\
\hline Cholangitis & 3.00 & $(1.21-7.41)$ & 0.02 \\
\hline Cholelithiasis & 1.64 & $(0.87-3.09)$ & 0.12 \\
\hline Hepatitis B & 0.81 & $(0.25-2.67)$ & 0.73 \\
\hline Hepatitis C & 0.64 & $(0.15-2.66)$ & 0.54 \\
\hline Chronic kidney disease & 0.74 & $(0.40-1.37)$ & 0.34 \\
\hline Any cardiovascular or cerebrovascular accident event & 0.93 & $(0.68-1.28)$ & 0.67 \\
\hline Atherosclerosis & 0.63 & $(0.16-2.53)$ & 0.51 \\
\hline \multicolumn{4}{|l|}{ Prior medications } \\
\hline a-blockers & 0.40 & $(0.06-2.86)$ & 0.36 \\
\hline Thiazide-type diuretics & 1.00 & $(0.65-1.53)$ & 0.99 \\
\hline B-blockers & 1.01 & $(0.71-1.45)$ & 0.95 \\
\hline Calcium channel blockers & 0.92 & $(0.67-1.26)$ & 0.60 \\
\hline Angiotensin-converting enzyme inhibitors & 0.88 & $(0.58-1.32)$ & 0.54 \\
\hline Angiotensin II receptor antagonists & 1.00 & $(0.73-1.37)$ & 0.99 \\
\hline \multicolumn{4}{|l|}{ HbAlc value } \\
\hline Lack of HbA1c data & 0.15 & $(0.02-1.09)$ & 0.06 \\
\hline $\mathrm{HbAlc}<7.0 \%$ & \multicolumn{3}{|l|}{ Ref } \\
\hline $7.0 \% \leq \mathrm{HbAlc}<8.5 \%$ & 1.18 & $(0.82-1.71)$ & 0.38 \\
\hline $\mathrm{HbAlc} \geq 8.5 \%$ & 1.80 & $(1.30-2.50)$ & $<0.01$ \\
\hline \multicolumn{4}{|l|}{ Concomitant medications } \\
\hline \multicolumn{4}{|l|}{ Clopidogrel } \\
\hline Non-exposure & \multicolumn{3}{|l|}{ Ref } \\
\hline $1-90$ days & 1.06 & $(0.59-1.89)$ & 0.84 \\
\hline$>90$ days & 0.41 & $(0.22-0.75)$ & $<0.01$ \\
\hline \multicolumn{4}{|l|}{ Ticagrelor } \\
\hline Non-exposure & \multicolumn{3}{|l|}{ Ref } \\
\hline Yes & 1.44 & $(0.45-4.62)$ & 0.54 \\
\hline \multicolumn{4}{|l|}{ Other anti-platelet agents } \\
\hline Non-exposure & \multicolumn{3}{|l|}{ Ref } \\
\hline Yes & 0.98 & $(0.57-1.71)$ & 0.96 \\
\hline \multicolumn{4}{|l|}{$\mathrm{H}_{2}$-blocker or proton pump inhibitors } \\
\hline Non-exposure & \multicolumn{3}{|l|}{ Ref } \\
\hline $1-4$ days & 0.73 & $(0.41-1.29)$ & 0.28 \\
\hline 5-30 days & 0.51 & $(0.33-0.79)$ & $<0.01$ \\
\hline $31-90$ days & 0.27 & $(0.15-0.48)$ & $<0.01$ \\
\hline$>90$ days & 0.30 & $(0.21-0.43)$ & $<0.01$ \\
\hline Metformin & & & \\
\hline Non-exposure & Ref & & \\
\hline Continued & & & \\
\hline
\end{tabular}




\begin{tabular}{|l|l|l|l|}
\hline & Adjusted HR & $\mathbf{9 5} \% \mathrm{CI}$ & $\boldsymbol{p}$ value \\
\hline $1-30$ days & 0.96 & $(0.55-1.67)$ & 0.89 \\
\hline $31-90$ days & 0.62 & $(0.34-1.14)$ & 0.12 \\
\hline$>90$ days & 0.50 & $(0.35-0.72)$ & $<0.01$ \\
\hline
\end{tabular}

Table 4. Risk of K. pneumoniae liver abscess adjusted for in-hospital mortality as a competing risk. CI, confidence interval; HbA1c, glycated hemoglobin; HR, hazard ratio. ${ }^{\star}$ Due to the small number of patients, unspecified liver injury, other viral hepatitis (exclude hepatitis B and hepatitis C), and centrally acting antiadrenergic agents were not considered in the analyses.

In conclusion, we found that females, taking clopidogrel or metformin for $\geq 90$ days, and taking H2-blockers or proton pump inhibitors for $\geq 5$ days, and taking aspirin $>90$ days were associated a reduced risk of KP-PLA in diabetic patients without increasing mortality. In contrast, aspirin had no effect on invasive KP-PLA syndrome in these patients. Further randomized controlled trial might be required to establish the role of aspirin in prevention of KP-PLA in diabetic patients.

\section{Methods}

Data source. The present retrospective cohort study was performed using the Chang Gung Research Database (CGRD), which is an electronic health records dataset from a large healthcare delivery system at Chang Gung Memorial Hospital in Taiwan, which includes two medical centers and five local hospitals ${ }^{38}$. The CGRD covers approximately $10 \%$ of all health services within the Taiwan National Health Insurance program, which is a single-payer nationwide health insurance program that covers over $99 \%$ of Taiwan's population ${ }^{39}$. The CGRD contains detailed diagnostic, prescription, and laboratory test result information from the emergency department, inpatient, and outpatient settings. The current study was approved by the Institutional Review Board of Chang Gung Medical Foundation, Taipei, Taiwan (201900920B0). All personal identifiable information was anonymized, and, therefore, the need for informed consent was waived. All methods were carried out in accordance with relevant guidelines and regulations.

Study cohort. We identified patients from the CGRD who had DM and were being newly treated with antidiabetic therapy (ADT) (i.e., insulin, GLP-1 agonists or oral hypoglycemic agents) between January 1st 2001 and December 31st 2018. The information of the diabetic patients prescribed ADT was retrieved from the CGRD via their Anatomical Therapeutic Chemical (ATC) codes in the outpatient setting (Supplementary Table S2). The date the patients were first prescribed ADT was defined as the date they received the diagnosis of DM. Patients were excluded if they were aged $<20$ years at the date of their diagnosis of DM; or were diagnosed with amebic liver abscess and PLA in the same hospitalization; had a prior hospitalization for PLA; had a history of aspirin use; or had no hospital admission or visit within a one-year prior to their diagnosis (Fig. 1). In addition, for outcome measurements, only diabetic patients who had at least one hospitalization during the follow-up were included in the study cohort.

Included patients were classified into aspirin users and non-users. Aspirin users were defined as those who started taking aspirin following the date of their diabetes diagnosis, and the date of their first aspirin prescription was defined as the study index date. Propensity-score matching (PSM) with a 1:1 ratio was employed to balance the distribution of baseline characteristics between aspirin users and non-users who were alive at the time aspirin was first prescribed to their matched users. The details of the PSM approach are described in the following statistical analysis section.

Outcomes. The primary outcome of interest was KP-PLA, as defined by an International Classification of Disease, 9th and 10th Clinical Modification (ICD-9/10-CM) code for liver abscess at the time of hospital discharge (Supplementary Table S3). KP infection was defined as having a blood, pus or abscess culture that yielded the growth of KP during the hospital stay. The secondary outcome was invasive KP-PLA syndrome, which was a subgroup of KP-PLA, defined as KP-PLA with a metastatic infection, such as endophthalmitis, brain abscess, intra-spinal abscess, extradural and subdural abscess, bacterial meningitis, lung abscess, osteomyelitis, or an abscess of the prostate (Supplementary Table S3) ${ }^{12}$. Any cause of in-hospital mortality was assessed in the analysis. Every patient was followed from the study index date until an event of interest occurred, loss to follow-up, or the last date of the dataset (December 31st, 2018), whichever occurred first.

Covariates. Baseline infection risk, age, sex, co-morbidities including liver cirrhosis, unspecified liver injury, hepatobiliary malignancy, gastric cancer, colon cancer, hepatocellular carcinoma, metastatic cancers, other malignancies, cholangitis, cholelithiasis, hepatitis B, hepatitis C, other viral hepatitis, chronic kidney disease, ischemic heart disease and myocardial infarction, ischemic stroke, arrythmia, any cardiovascular or cerebrovascular disease, or atherosclerosis were identified using the ICD-9/10-CM codes from outpatient visits or hospital discharges within 1 year prior to the study index date (Supplementary Table S3).

Prior medical use of centrally acting anti-adrenergic agents, alpha- and beta-blockers, thiazide-type diuretics, calcium channel blockers, angiotensin-converting enzyme inhibitors, and angiotensin II receptor antagonists within 1 year prior to the index date was identified by ATC codes (Supplementary Table S2). In addition, 
anti-platelet agents (clopidogrel, ticagrelor and others), $\mathrm{H} 2$ receptor antagonists (H2-blockers), or proton pump inhibitors prescribed during the study follow-up were considered as concomitant medications (Supplementary Table S2).

Statistical analysis. Continuous variables were reported as the mean and standard deviation (SD) and categorical variables were reported as the number and percentage. The individual's propensity-score was calculated using multivariate logistic regression, including class of ADT $(n=9)$, baseline comorbid conditions, prior medications, glycated hemoglobin, and interval between diabetes diagnosis and first aspirin prescription (39 variables in total). To minimize the possible effect of immortal time bias, the length of time from diabetes diagnosis to the index date (aspirin initiation) was ascertained and randomly selected non-users who were followed during the same period. The balance between aspirin user and non-user groups was assessed using the absolute standardized mean differences of individual covariates at baseline before and after matching, using a threshold of $<0.1$ to indicate balance.

The cumulative incidence of an event of interest between the two groups was analyzed using the Kaplan-Meier approach with a log-rank test. Death-censored Cox proportional hazards regression was used to estimate the adjusted hazard ratio (aHR) with 95\% confidence intervals (CIs) for KP-PLA or invasive KP-PLA syndrome in the PSM cohort. The level of significance was set as $5 \%(p<0.05)$ for two-sided tests. Statistical analyses and data management were performed using SAS software version 9.4 (SAS Institute Inc. Cary, NC, USA).

\section{Data availability}

The datasets generated during and/or analyzed during the current study are available from the corresponding author on reasonable request.

Received: 26 August 2020; Accepted: 17 November 2020

Published online: 07 December 2020

\section{References}

1. Chen, Y. C., Lin, C. H., Chang, S. N. \& Shi, Z. Y. Epidemiology and clinical outcome of pyogenic liver abscess: an analysis from the National Health Insurance Research Database of Taiwan, 2000-2011. J. Microbiol. Immunol. Infect. 49, 646-653 (2016).

2. Meddings, L. et al. A population-based study of pyogenic liver abscesses in the United States: incidence, mortality, and temporal trends. Am. J. Gastroenterol. 105, 117-124 (2010).

3. Jepsen, P., Vilstrup, H., Schonheyder, H. C. \& Sorensen, H. T. A nationwide study of the incidence and 30-day mortality rate of pyogenic liver abscess in Denmark, 1977-2002. Aliment Pharmacol. Ther. 21, 1185-1188 (2005).

4. Molle, I., Thulstrup, A. M., Vilstrup, H. \& Sorensen, H. T. Increased risk and case fatality rate of pyogenic liver abscess in patients with liver cirrhosis: a nationwide study in Denmark. Gut 48, 260-263 (2001).

5. Tian, L. T. et al. Liver abscesses in adult patients with and without diabetes mellitus: an analysis of the clinical characteristics, features of the causative pathogens, outcomes and predictors of fatality: a report based on a large population, retrospective study in China. Clin. Microbiol. Infect. 18, E314-330 (2012).

6. Ko, M. C. et al. A cohort study of age and sex specific risk of pyogenic liver abscess incidence in patients with type 2 diabetes mellitus. Medicine (Baltimore) 98, 15366 (2019).

7. Keynan, Y. \& Rubinstein, E. Diabetes mellitus and pyogenic liver abscess: risk and prognosis. Clin. Infect. Dis. 45, 801 (2007).

8. Li, W., Chen, H., Wu, S. \& Peng, J. A comparison of pyogenic liver abscess in patients with or without diabetes: a retrospective study of 246 cases. BMC Gastroenterol. 18, 144 (2018).

9. Fung, C. P. et al. A 5-year study of the seroepidemiology of Klebsiella pneumoniae: high prevalence of capsular serotype K1 in Taiwan and implication for vaccine efficacy. J. Infect. Dis. 181, 2075-2079 (2000).

10. Lin, J. C. et al. High prevalence of phagocytic-resistant capsular serotypes of Klebsiella pneumoniae in liver abscess. Microbes Infect. 6, 1191-1198 (2004).

11. Zhang, S. et al. Clinical, microbiological, and molecular epidemiological characteristics of Klebsiella pneumoniae-induced pyogenic liver abscess in southeastern China. Antimicrob. Resist. Infect. Control 8, 166 (2019).

12. Siu, L. K., Yeh, K. M., Lin, J. C., Fung, C. P. \& Chang, F. Y. Klebsiella pneumoniae liver abscess: a new invasive syndrome. Lancet Infect. Dis. 12, 881-887 (2012).

13. Lee, I. R. et al. Comparison of diabetic and non-diabetic human leukocytic responses to different capsule types of klebsiella pneumoniae responsible for causing pyogenic liver abscess. Front. Cell. Infect. Microbiol. 7, 401 (2017).

14. Zhang, M. et al. The NFkappaB signaling pathway serves an important regulatory role in Klebsiella pneumoniae liver abscesses. Exp. Ther. Med. 15, 5443-5449 (2018).

15. Li, B., Zhao, Y., Liu, C., Chen, Z. \& Zhou, D. Molecular pathogenesis of Klebsiella pneumoniae. Future Microbiol. 9, 1071-1081 (2014).

16. Lee, C. H. et al. Impact of glycemic control on capsular polysaccharide biosynthesis and opsonophagocytosis of Klebsiella pneumoniae: implications for invasive syndrome in patients with diabetes mellitus. Virulence 7, 770-778 (2016).

17. Lin, J. C. et al. Impaired phagocytosis of capsular serotypes K1 or K2 Klebsiella pneumoniae in type 2 diabetes mellitus patients with poor glycemic control. J. Clin. Endocrinol. Metab. 91, 3084-3087 (2006).

18. Liu, J. S. et al. Aspirin use is associated with reduced risk for recurrence of pyogenic liver abscess: a propensity score analysis. Sci. Rep. 9, 11511 (2019).

19. Prescott, D. \& McKay, D. M. Aspirin-triggered lipoxin enhances macrophage phagocytosis of bacteria while inhibiting inflammatory cytokine production. Am. J. Physiol. Gastrointest. Liver Physiol. 301, G487-497 (2011).

20. Lee, C. H. et al. Aspirin enhances opsonophagocytosis and is associated to a lower risk for Klebsiella pneumoniae invasive syndrome. BMC Infect. Dis. 14, 47 (2014).

21. Domenico, P., Schwartz, S. \& Cunha, B. A. Reduction of capsular polysaccharide production in Klebsiella pneumoniae by sodium salicylate. Infect. Immun. 57, 3778-3782 (1989).

22. Lee, C. H., Chuah, S. K., Tai, W. C. \& Chen, I. L. Platelet reactivity in diabetic patients with invasive Klebsiella pneumoniae liver abscess syndrome. Infect. Drug Resist. 11, 1669-1676 (2018).

23. Chao, G., Ye, F., Shen, W., Gong, W. \& Zhang, S. Study on the characteristic of intestinal flora in patients with dual antiplatelet therapy. J. Drug Target. 28, 500-507 (2020).

24. Chen, N. et al. Altered profiles of gut microbiota in Klebsiella pneumoniae-induced pyogenic liver abscess. Curr. Microbiol. 75, 952-959 (2018). 
25. Mogna, L. et al. In Vitro Inhibition of Klebsiella pneumoniae by Lactobacillus delbrueckii Subsp. delbrueckii LDD01 (DSM 22106): an innovative strategy to possibly counteract such infections in humans?. J. Clin. Gastroenterol. 50(Suppl 2), S136-S139 (2016).

26. Gommers, L. M. M. et al. Low gut microbiota diversity and dietary magnesium intake are associated with the development of PPI-induced hypomagnesemia. FASEB J. 33, 11235-11246 (2019).

27. Jackson, M. A. et al. Proton pump inhibitors alter the composition of the gut microbiota. Gut 65, 749-756 (2016).

28. Gupta, R. W. et al. Histamine-2 receptor blockers alter the fecal microbiota in premature infants. J. Pediatr. Gastroenterol. Nutr. 56, 397-400 (2013).

29. Shindo, K., Yamazaki, R., Koide, K., Fukumura, M. \& Hirai, Y. Alteration of bile acid metabolism by cimetidine in healthy humans. J. Investig. Med. 44, 462-469 (1996).

30. Wang, Y. P., Liu, C. J., Chen, T. J., Lin, Y. T. \& Fung, C. P. Proton pump inhibitor use significantly increases the risk of cryptogenic liver abscess: a population-based study. Aliment Pharmacol. Ther. 41, 1175-1181 (2015).

31. Delcaru, C. et al. Antagonistic activities of some Bifidobacterium sp. strains isolated from resident infant gastrointestinal microbiota on Gram-negative enteric pathogens. Anaerobe 39, 39-44 (2016)

32. Wu, H. et al. Metformin alters the gut microbiome of individuals with treatment-naive type 2 diabetes, contributing to the therapeutic effects of the drug. Nat. Med. 23, 850-858 (2017).

33. Rodriguez, J., Hiel, S. \& Delzenne, N. M. Metformin: old friend, new ways of action-implication of the gut microbiome?. Curr. Opin. Clin. Nutr. Metab. Care 21, 294-301 (2018).

34. Zhang, M. et al. Effects of metformin, acarbose, and sitagliptin monotherapy on gut microbiota in Zucker diabetic fatty rats. BMJ Open Diabetes Res. Care 7, e000717 (2019).

35. Shi, S. H., Zhai, Z. L. \& Zheng, S. S. Pyogenic liver abscess of biliary origin: the existing problems and their strategies. Semin. Liver Dis. 38, 270-283 (2018).

36. Lin, Y. T., Wang, F. D., Wu, P. F. \& Fung, C. P. Klebsiella pneumoniae liver abscess in diabetic patients: association of glycemic control with the clinical characteristics. BMC Infect. Dis. 13, 56 (2013).

37. Tsai, M. S. et al. Chang Gung Research Database: A multi-institutional database consisting of original medical records. Biomed. J. 40, 263-269 (2017).

38. Shao, S. C. et al. The Chang Gung Research Database-A multi-institutional electronic medical records database for real-world epidemiological studies in Taiwan. Pharmacoepidemiol. Drug Saf. 28, 593-600 (2019).

39. Hsieh, C. Y. et al. Taiwan's National Health Insurance Research Database: past and future. Clin Epidemiol 11, 349-358 (2019).

\section{Acknowledgments}

The authors would like to thank Ms. I-Shiuan Tsai at the Biostatistics Center, Kaohsiung Chang Gung Memorial Hospital for her assistance with the data management and statistical analyses. We also thank Dr. Chien-Ching Hung at the Department of Internal Medicine, National Taiwan University Hospital, for his critical review of this manuscript.

\section{Author contributions}

Conceptualization: C.H.T., C.N.H., S.C.Y., C. K.W., C.M.L., W.C.T., S.K.C., C.H.L. Data collection: C.N.H. Data analysis and interpretation: C.H.T., C.N.H., C.H.L. Writing-original draft: C.H.T. Writing-review \& editing: C.H.L.

\section{Funding}

This study was supported by the Research Foundation of Kaohsiung Chang Gung Memorial Hospital (CFRPG8J0071).

\section{Competing interests}

The authors declare no competing interests.

\section{Additional information}

Supplementary information is available for this paper at https://doi.org/10.1038/s41598-020-78442-8.

Correspondence and requests for materials should be addressed to C.-H.L.

Reprints and permissions information is available at www.nature.com/reprints.

Publisher's note Springer Nature remains neutral with regard to jurisdictional claims in published maps and institutional affiliations.

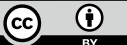

Open Access This article is licensed under a Creative Commons Attribution 4.0 International License, which permits use, sharing, adaptation, distribution and reproduction in any medium or format, as long as you give appropriate credit to the original author(s) and the source, provide a link to the Creative Commons licence, and indicate if changes were made. The images or other third party material in this article are included in the article's Creative Commons licence, unless indicated otherwise in a credit line to the material. If material is not included in the article's Creative Commons licence and your intended use is not permitted by statutory regulation or exceeds the permitted use, you will need to obtain permission directly from the copyright holder. To view a copy of this licence, visit http://creativecommons.org/licenses/by/4.0/.

(C) The Author(s) 2020 